\title{
Aggregatibacter actinomycetemcomitans osteomyelitis in a 12 year old boy: case report emphasizing the importance of tissue culture, and review of literature
}

\author{
Ketaki Sharma', Poonam Mudgil ${ }^{1 *}$, John S. Whitehall ${ }^{1}$ and lain Gosbell ${ }^{2}$
}

\begin{abstract}
Background: Aggregatibacter actinomycetemcomitans most commonly causes periodontitis but has been reported to infect heart valves, soft tissue, brain and lungs, and distal bones. Osteomyelitis distal to the jaw is rarely described.

Case presentation: We report an unusual and rare case of chronic osteomyelitis caused by A. actinomycetemcomitans in the toe of a paediatric patient, and review the available literature. The infection was managed with intravenous antibiotics followed by oral antibiotics.

Conclusion: This is an unusual presentation of A. actinomycetemcomitans causing chronic osteomyelitis presumed due to nidation in a minimally damaged bone, associated with bacteraemia of an oral commensal. It occurred in the toe, without obvious dental predisposition; associated with minimal clinical disturbance and with muted immune response.
\end{abstract}

\section{Background}

Aggregatibacter actinomycetemcomitans, previously known as Actinobacillus actinomycetemcomitans, is a microaerophilic, capnophilic gram negative cocco-bacillus that is an oral commensal in over $30 \%$ of apparently healthy children [1]. It is most commonly associated with aggressive periodontitis but that infection may be chronic [2]. Extra-oral infections, presumably due to haematogenous spread from infected periodontium [3], have occurred on heart valves and in soft tissue including brain and lung, joints and distal bones, mostly in adults. We report distal, chronic osteomyelitis in a 12 year old boy.

\section{Case presentation}

An otherwise well, immunocompetent 12 year old boy presented to Campbelltown Hospital, NSW, with a 2 week history of pain, swelling, and purulent discharge

\footnotetext{
*Correspondence: p.mudgil@westernsydney.edu.au

${ }^{1}$ Department of Paediatrics, School of Medicine, Western Sydney

University, Sydney, NSW, Australia

Full list of author information is available at the end of the article
}

from his right great toe. His past dental history is unknown and not recorded in records. He had 'stubbed' the toe 12 months previously and mild, intermittent pain had persisted, un-associated with any systemic symptoms or signs of disease. Two weeks before presentation, a purulent discharge had emerged from the side of the toe. He had received a course of oral flucloxacillin from his general practitioner. On examination, there was superficial crusting on the medial aspect of the right great toe associated with purulent discharge. There was mild swelling around the inter-phalangeal joint but no erythema and tenderness, and there was full and painless range of movement throughout the foot. There were no abnormalities found on general examination of the child, and his teeth and gums appeared normal on inspection of his mouth. Subsequent dental review revealed no evidence of periodontitis.

Investigations revealed a normal white cell count (total $6.2 \times 10^{9} / \mathrm{L}$; neutrophils $\left.1.96 \times 10^{9} / \mathrm{L}\right)$, un-elevated levels of inflammatory markers (erythrocyte sedimentation rate $2 \mathrm{~mm} / \mathrm{h}$, C-reactive protein $<0.4 \mathrm{mg} / \mathrm{L})$, and both 
gram positive cocci and gram negative bacilli in a wound swab whose culture failed to grow organisms. An X-ray revealed a lytic lesion in the proximal phalanx of the great toe while bone scan confirmed cystic changes, sclerosis and hyperaemia (Fig. 1).

At operation, a sinus was found passing through organised purulent material to the infected proximal end of the phalanx. There was no sequestered bone. After 6 days, culture of curetted bone grew A. actinomycetemcomitans which was sensitive to ampicillin and cefotaxime. According to the Sydney South West Pathology Service tissue protocol, culturing is done on horse blood, chocolate, MacConkey, Columbia colistin nalidixic acid (CAN), and anaerobic agars, and thioglycollate broth. Incubation is done at $37{ }^{\circ} \mathrm{C}$ and read daily for up to 7 days before reported as final culture negative. A blood culture had been taken at presentation and was negative at 5 days.

His treatment had begun with intravenous flucloxacillin and clindamycin but the latter was ceased and cefotaxime started when culture results became available. A total of 7 days of intravenous therapy was followed by 12 weeks of oral amoxicillin during which the infection resolved. Given the known association of A. actinomycetemcomitans infections with endocarditis, an echocardiogram was performed but no abnormalities were detected.

\section{Discussion}

Aggregatibacter actinomycetemcomitans is a member of the Haemophilus aphrophilus now named Aggregatibacter aphrophilus, Actinobacillus actinomycetemcomitans, Cardiobacterium hominis, Eikenella corrodens and Kingella spp. (HACEK) group of gram negative bacteria

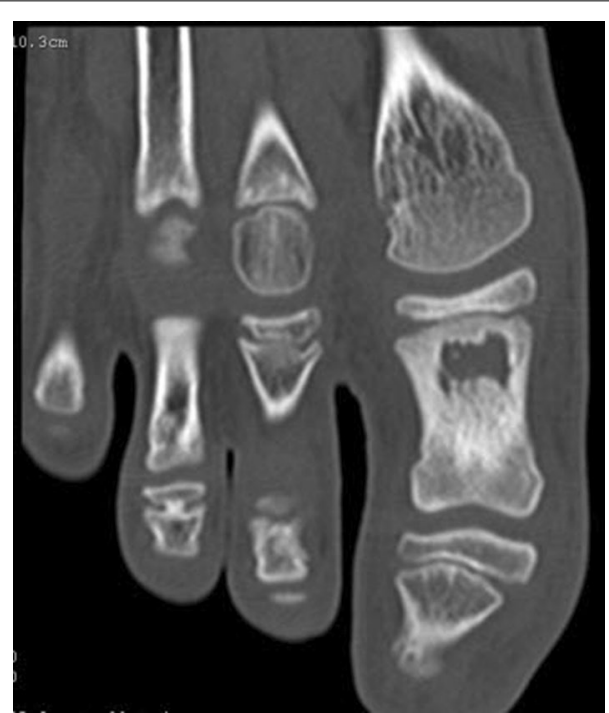

Fig. 1 X-ray image showing lytic changes of osteomyelitis in the proximal phalanx of the big toe characterized by fastidious, capnophilic growth requirements. Kingella kingae is a well-recognised albeit rare cause of skeletal infections in children. Conversely, $A$. actinomycetemcomitans is noted for its association with both aggressive and chronic periodontal infection, as well as for rare infection of heart valves and soft tissue including brain and lungs. While infection of the bone of the jaw may be an extension of periodontitis, osteomyelitis of distal bones is most rare, and usually involves the vertebrae.

A medline bibliographic search was conducted to identify documented cases of distal osteomyelitis caused by A. actinomycetemcomitans, in both its recent and former names. Data was collected to include age, sex, clinical presentation, time to diagnosis, site of infection, laboratory findings and predisposing factors and these details together with those of our patient are presented in Table 1.

There were seven bone infections due to A. actinomycetemcomitans, and one with an organism apparently intermediate between it and Haemophilus aphrophilus. In two of these eight cases, there was co-infection of $A$. actinomycetemcomitans with other bacteria, which is not unusual [4]. The age of the patients ranged from 16 to 78 years; seven were male.

Four infections involved vertebrae, which is unsurprising given they are the most common site of acute haematogenous osteomyelitis in adults. Six infections were associated with dental disease or oral trauma: four had dental disease, one a mandibular fracture, and one had an opponent's tooth embedded in his hand. One patient had septic arthritis of the knee and chronic osteomyelitis of the distal femur, but had had an open fracture of the knee 20 years previously.

Duration of symptoms ranged from 2 days to 7 months, but three of the infections were classified as chronic osteomyelitis. The white cell count was normal to barely raised in all cases, but inflammatory markers were elevated in all, except our patient. Resistance to antibiotics was not reported.

The infectivity of $A$. actinomycetemcomitans is due to many virulence factors that modulate host immunity, facilitate invasion and retard repair. Exotoxins include a leukotoxin (LtxA) which promotes lysis of defending macrophages, mast cells and leukocytes, and a cytolethal distending toxin (CDT) which promotes death of host cells by restricting their proliferation while encouraging osteoclastogenesis. The exotoxin leukotoxin A combines with endotoxins to induce inflammation with subsequent bone resorption $[3,5]$. Defence of the organism is aided by the formation of biofilms with synergistic organisms in the protected crevice between the gums and the teeth. The film can attach to the teeth and be nourished by a 


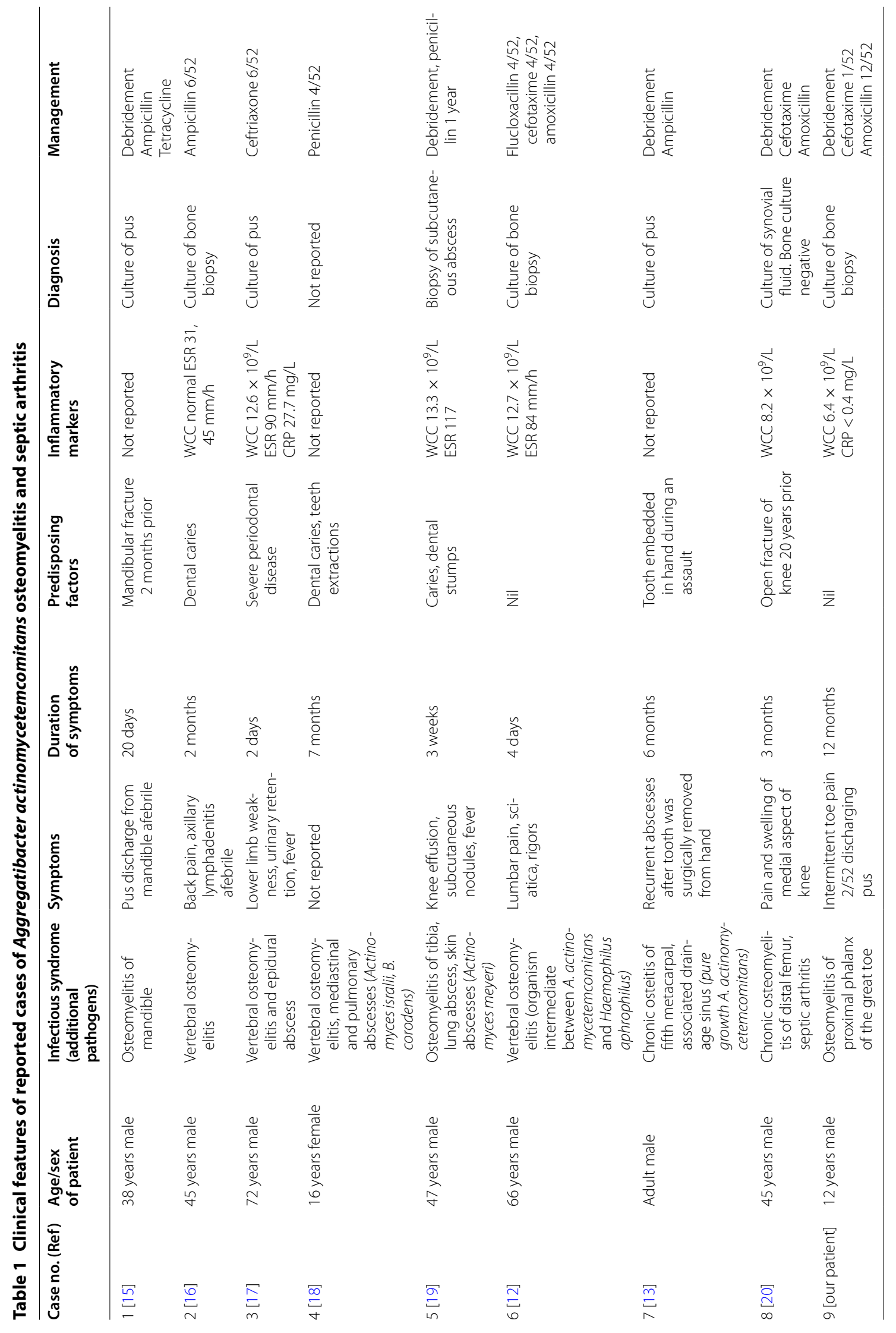


continuous efflux of fluid derived from blood plasma and containing amino acids, peptides and proteins [6].

Aggregatibacter actinomycetemcomitans has been found in the mouths of $>1 / 3$ of apparently healthy children and is believed to travel horizontally and vertically within families, presumably spread in saliva $[7,8]$. A recently reported case of chronic wound infection suggests an exogenous origin [9] but, otherwise, infections distal to the mouth appear to be endogenous and blood borne. Periodontal disease is believed to predispose to such spread. It should be noted that periodontal disease (gingivitis associated with loss of the tooth-supporting tissues, alveolar bone and connective tissues) may not be associated with marked evidence of dental caries. Indeed, a negative relationship has been described [10].

Osteomyelitis in hands and feet is unusual in children unless associated with dactylitis of a haemoglobinopathy in which cases there is acute onset with pain, swelling and, usually, systemic symptoms associated with salmonellae infection [11]. Chronic, symptom-minimal infection is rare but may occur with tuberculosis [12].

We can only postulate about the lack of aggression by $A$. actinomycetemcomitans and the muted immune response in our patient: perhaps the organism entered a sessile state of biofilm existence while deprived of nutritionally rich gingival crevicular fluid [13] and also deprived of synergistic relationships with other oral commensals [14].

Knowing that most bone infections in children are due to $S$. aureus, treatment of our patient was started with flucloxacillin, which was continued for 1 week. After isolation of $A$. actinomycetemcomitans, the flucloxacillin was changed to amoxicillin, since HACEK organisms are not susceptible to flucloxacillin, and the isolate was found to be susceptible to amoxicillin The gram positive cocci and gram negative rods seen on gram stain from the wound swab did not grow in the blood culture, and may have been anaerobes, which are commonly found with organisms such as A. actinomycetemcomitans. Alternatively the prior antibiotics from the family doctor might have inhibited the growth of bacteria. Resistance to amoxicillin has been reported in $77 \%$ of distal infections or cultures of A. actinomycetemcomitans derived from the mouth, though sensitivity to amoxicillin/clavulanic acid is expected [15].

\section{Conclusion}

We have described an unusual presentation of A. actinomycetemcomitans osteomyelitis presumed due to nidation in a minimally damaged bone, associated with bacteraemia of an oral commensal. It occurred in the big toe of a young, otherwise healthy child without obvious dental predisposition; was chronic and associated with minimal clinical disturbance until a sinus opened to the skin; was unassociated with alteration in white cell count and inflammatory markers and was sensitive to amoxicillin. The peripheral wound swab did not grow $A$. actinomycetemcomitans. Representative specimens are important, especially if blood cultures are negative and/ or there is failure of empirical treatment. Surface swabs can be misleading, and deep specimens obtained by needle or surgery are preferred to diagnose the cause of bone infection.

\section{Authors' contributions}

KS collected data and did most writing, JSW ideated the case report, IG and PM helped in drafting the manuscript. All authors read and approved the final manuscript.

\section{Author details}

${ }^{1}$ Department of Paediatrics, School of Medicine, Western Sydney University, Sydney, NSW, Australia. ${ }^{2}$ Department of Infectious Diseases and Microbiology, School of Medicine, Western Sydney University, Sydney, NSW, Australia.

\section{Author's information}

KS, PM, JSW: Department of Paediatrics, School of Medicine, Western Sydney University, New South Wales, Australia; IG: Department of Infectious Diseases and Microbiology,School of Medicine, Western Sydney University, New South Wales, Australia; Antibiotic Resistance and Mobile Elements Group, Ingham Institute for Applied Medical Research, Liverpool, NSW, Australia; Molecular Medicine Research Group, School of Medicine,Western Sydney University, NSW, Australia; and Department of Microbiology and Infectious Diseases, Sydney South West Pathology Service (Liverpool), NSW Health Pathology, NSW, Australia

\section{Acknowledgements}

Not applicable.

Competing interests

The authors declare that they have no competing interests.

Availability of data and materials

All data generated or analysed during this study are included in this article.

Consent for publication

Written informed consent was obtained from the patient's parent for publication of this case report.

Received: 5 October 2016 Accepted: 6 March 2017

Published online: 14 March 2017

\section{References}

1. Lamell CW, Griffen AL, McClellan DL, Leys EJ. Acquisition and colonization stability of Actinobacillus actinomycetemcomitans and Porphyromonas gingivalis in children. J Clin Microbiol. 2000;38:1196-9.

2. Erriu M, Pili FM, Tuveri E, Pigliacampo D, Scano A, Montaldo C, Piras V, Denotti G, Pilloni A, Garau V, Orrù G. Oil essential mouthwashes antibacterial activity against Aggregatibacter actinomycetemcomitans: a comparison between antibiofilm and antiplanktonic effects. Int J Dent. 2013;2013:164267.

3. Raja M, Ummer F, Dhivakar CP. Aggregatibacter actinomycetemcomitansa tooth killer. J Clin Diagn Res. 2014;8:ZE13-6.

4. Antony B, Thomas S, Chandrashekar SC, Kumar MS, Kumar V. Osteomyelitis of the mandible due to Aggregatibacter (Actinobacillus) actinomycetemcomitans. Indian J Pathol Microbiol. 2009:52:115-6.

5. Muhle I, Rau J, Ruskin J. Vertebral osteomyelitis due to Actinobacillus actinomycetemcomitans. JAMA. 1979;241:1824-5. 
6. Patel SM, Mo JH, Walker MT, Adley B, Noskin GA. Epidural abscess and osteomyelitis due to Actinobacillus actinomycetemcomitans. Diagn Microbiol Infect Dis. 2004;50:283-5.

7. Kaplan AH, Weber DJ, Oddone EZ, Perfect JR. Infection due to Actinobacillus actinomycetemcomitans: 15 cases and review. Rev Inf Dis. 1989:11:46-63.

8. Apotheloz C, Regamey C. Disseminated infection due to Actinomyces meyeri: case report and review. Clin Infect Dis. 1996;22:621-5.

9. Farrington M, Eykyn SJ, Walker M, Warren RE. Vertebral osteomyelitis due to coccobacilli of the HB group. Br Med J. 1983;287:1658-60.

10. Mauff AC, Miller S, Kuhnle V, Carmichael M. Infections due to Actinobacillus actinomycetemcomitans. A report of 3 cases. S Afr Med J. 1983;63:580-1.

11. Molina F, Echániz A, Durán MT, Diz-Lois F. Infectious arthritis of the knee due to Actinobacillus actinomycetemcomitans. Eur J Clin Microbiol Infect Dis. 1994;13:687-9.

12. Rahamat-Langendoen JC, van Vonderen MG, Engström LJ, Manson WL, van Winkelhoff AJ, Mooi-Kokenberg EA. Brain abscess associated with Aggregatibacter actinomycetemcomitans: case report and review of literature. J Clin Periodontol. 2011;38:702-6.

13. Henderson B, Ward JM, Ready D. Aggregatibacter (Actinobacillus) actinomycetemcomitans: a triple $A^{*}$ periodontopathogen? Periodontology. 2000;201054:78-105.

14. Åberg CH, Kelk P, Johansson A. Aggregatibacter actinomycetemcomitans: virulence of its leukotoxin and association with aggressive periodontitis. Virulence. 2015;6:188-95.
15. Van Winkelhoff AJ, Boutaga K. Transmission of periodontal bacteria and models of infection. J Clin Periodontol. 2005;32(Suppl 6):16-27.

16. Doğan B, Kipalev AS, Okte E, Sultan N, Asikainen SE. Consistent intrafamilial transmission of Actinobacillus actinomycetemcomitans despite clonal diversity. J Periodontol. 2008;79:307-15.

17. Böhme J, Glaenz TE, Kaiser A. Allmacher C [Cutaneous infection with Aggregatibacter actinomycetemcomitans]. Hautarzt. 2014;65:810-3.

18. Ramachandra SS. Low levels of caries in aggressive periodontitis: a literature review. Saudi Dent J. 2014:26:47-9.

19. Bennett OM. Salmonella osteomyelitis and the hand-foot syndrome in sickle cell disease. J Pediatr Orthop. 1992;12:534-8.

20. Stacy A, Everett J, Jorth P, Trivedi U, Rumbaugh KP, Whiteley M. Bacterial fight-and-flight responses enhance virulence in a polymicrobial infection. Proc Natl Acad Sci. 2014;111:7819-24.

21. Street $M$, Puna R, Huang M, Crawford H. Pediatric acute hematogenous osteomyelitis. J Pediatr Orthop. 2015:35(6):634-9.

22. van Winkelhoff AJ, Rurenga P, Singadji Z, Wekema-Mulder G. Metronidazole and amoxicillin susceptibility of Aggregatibacter actinomycetemcomitans. Int J Antimicrob Agents. 2014;43:475-6.

23. Lew DP, Waldvogel FA. Osteomyelitis. Lancet. 2004:364:369-79.

24. Lazzarini L, Lipsky BA, Mader JT. Antibiotic treatment of osteomyelitis: what have we learned from 30 years of clinical trials? Int J Infecti Dis. 2005:9:127-38.

25. Uçkay I, Jugun K, Gamulin A, Wagener J, Hoffmeyer P, Lew D. Chronic osteomyelitis. Curr Infect Dis Rep. 2012;14:566-75.

\section{Submit your next manuscript to BioMed Central and we will help you at every step:}

- We accept pre-submission inquiries

- Our selector tool helps you to find the most relevant journal

- We provide round the clock customer support

- Convenient online submission

- Thorough peer review

- Inclusion in PubMed and all major indexing services

- Maximum visibility for your research

Submit your manuscript at www.biomedcentral.com/submit 